\title{
Tecnologias de informação e comunicação para formação profissional em Odontologia: uma proposta do Telessaúde/ES
}

\author{
Karina Tonini dos Santos Pacheco*; Raniery Mattedi Nascimento**; Maria Zilma Rios***; Antonio \\ Carlos Pacheco Filho****; Carmen Barreira-Nielsen*****; Cléa Adas Saliba Garbin ******
}

\author{
* Doutora em Odontologia Preventiva e Social, Professora do \\ Departamento de Medicina Social, Universidade Federal do \\ Espírito Santo \\ ** Cirurgiã-dentista, Pesquisadora, Universidade Federal do Espírito \\ Santo \\ *** Mestre em História, Coordenadora da Teleducação, \\ Telesssaúde/ES, Universidade Federal do Espírito Santo \\ **** Cirurgião-dentista, Doutorando do Programa de Pós-Graduação \\ em Odontologia Preventiva e Social da Universidade Estadual \\ Paulista \\ ****** Doutora em Ciências Fisiológicas, Coordenadora do \\ Telessaúde/ES, Universidade Federal do Espírito Santo \\ ****** Professora Titular do Programa de Pós-Graduação em \\ Odontologia Preventiva e Social da Universidade Estadual \\ Paulista
}

Recebido em 26/10/2017. Aprovado em 22/07/2018.

\begin{abstract}
RESUMO
O Telessaúde/ES, com o auxílio de Tecnologias de Informação e Comunicação (TIC), promove a ampliação da Educação Permanente à Saúde (EPS) aos profissionais atuantes nas equipes de Estratégia Saúde da Família (ESF) do estado do Espírito Santo. O presente estudo objetivou apresentar uma proposta de um ambiente virtual em Odontologia, no âmbito do Telessaúde/ES, proporcionando um espaço exclusivo de compartilhamento de informações às Equipes de Saúde Bucal (ESB). Trata-se de uma pesquisa-ação, método adequado para propor soluções para a melhoria do acesso e utilização das ferramentas do Telessaúde/ES pelas ESB mediante às necessidades identificadas. Em 2017, o Telessaúde Bucal iniciou suas atividades no Telessaúde/ES, disponibilizando os serviços de webpalestras, teleconsultorias assíncronas e síncronas e materiais oficiais da área, ofertados na última sexta-feira de cada mês. O ambiente virtual de Telessaúde Bucal apresentado estará incluso no espaço de "Programas Especiais", permitindo o acesso a qualquer serviço. O Telessaúde Bucal é um serviço que poderá promover conhecimento e troca de experiências para a efetivação da EPS, estimulando maior participação dos profissionais da Odontologia. Descritores: Telemedicina. Educação Continuada. Tecnologia da Informação. Odontologia.
\end{abstract}




\section{INTRODUÇÃO}

Uma das competências do Sistema Único de Saúde (SUS) é o ordenamento da formação de recursos humanos na Área da Saúde ${ }^{1}$ Nessa perspectiva, a Educação Permanente em Saúde (EPS), entendida como aprendizagem voltada ao trabalho, objetiva a incorporação do conhecimento adquirido ao cotidiano dos profissionais que atuam no SUS, transformando suas práticas de trabalho diárias $^{2}$.

Em 2007, o Ministério da Saúde (MS) desenvolveu o projeto-piloto do Telessaúde Brasil com modernas Tecnologias de Informação e Comunicação (TIC) para o desenvolvimento de atividades à distância, visando ao fortalecimento e à ampliação da EPS para os profissionais atuantes na Estratégia Saúde da Família (ESF). No ano de 2011, o projeto foi redefinido como Programa Nacional Telessaúde Brasil Redes, cujo propósito foi a promoção do fortalecimento da articulação das Redes de Atenção à Saúde (RAS), coordenadas pela Atenção Básica (AB) no âmbito do SUS ${ }^{3}$.

O Telessaúde Brasil Redes fornece os serviços de Teleconsultoria, síncrona e assíncrona, Telediagnóstico, Segunda Opinião Formativa e Teleducação ${ }^{3}$. Esses serviços promovem educação para o trabalhador na perspectiva de melhoria da qualidade do atendimento ao público e na redução dos encaminhamentos médicos e deslocamentos desnecessários de pacientes ${ }^{3-5}$.

De acordo com a Portaria $\mathrm{n}^{\mathrm{o}} 2.546$, de 27 de outubro de $2011^{3}$, os serviços de Teleconsultoria, Telediagnóstico, Segunda Opinião Formativa e a Teleducação são definidos como:

a) Teleconsultoria - consulta registrada e realizada entre trabalhadores, profissionais e gestores da Área da Saúde, por meio de instrumentos de telecomunicação bidirecional, com o fim de esclarecer dúvidas sobre procedimentos clínicos, ações de saúde e questões relativas ao processo de trabalho, podendo ser síncrona (teleconsultoria realizada em tempo real, geralmente por chat, web ou videoconferência) ou assíncrona (teleconsultoria realizada por meio de mensagens off-line);

b) Telediagnóstico - serviço autônomo que utiliza as TIC para realizar serviços de apoio ao diagnóstico à distância (geográfica e temporal);

c) Segunda Opinião Formativa - resposta sistematizada, construída com base em revisão bibliográfica, nas melhores evidências científicas e clínicas e no papel ordenador da Atenção Básica à Saúde, a perguntas originadas das teleconsultorias, selecionadas a partir de critérios de relevância e pertinência em relação às diretrizes do SUS; e

d) Teleducação - conferências, aulas e cursos, ministrados por meio da utilização das TIC.

Em 2010, foi implantado o Núcleo de Telessaúde no Estado do Espírito Santo (NUTES), com a participação da Secretaria de Estado do Espírito Santo (SESA), do Instituto Federal do Espírito Santo (IFES), Campus Vitória, e da Universidade Federal do Espírito Santo (UFES) ${ }^{4}$.

O Telessaúde/ES disponibiliza os seus serviços a todas as categorias profissionais atuantes no SUS. Em 2016, começou-se a planejar mais um serviço, voltado especificamente às Equipes de Saúde Bucal (ESB), pois, por meio de um levantamento de informações realizado em 2014, verificou-se que os cirurgiões-dentistas eram os profissionais que menos utilizavam os serviços ofertados pelo Telessaúde/ES.

A Teleodontologia, um campo integrante do Telessaúde, vem evoluindo com ênfase na teleducação interativa, na teleassistência e na produção de pesquisas multicêntricas ${ }^{6}$. Além disso, possui a capacidade de aprimoramento da qualidade educacional das ESB, pois oferece 
ferramentas e programas de Saúde Bucal e teleconsultorias com especialistas capacitados, além de ser um método economicamente viável de promoção de atenção em saúde à população ${ }^{7}$.

Considerando o exposto, o objetivo deste trabalho é apresentar e discutir uma proposta de ambiente virtual de aprendizagem direcionado à Odontologia, com o intuito de proporcionar um espaço exclusivo de compartilhamento de informações e conhecimentos visando ao fortalecimento da qualificação das ESB na Atenção Básica.

\section{METODOLOGIA}

Este estudo utiliza a metodologia denominada pesquisa-ação, cujo propósito é de modificar uma situação específica por meio de análises, reflexões e críticas das práticas, possibilitando a intervenção e modificação dessa dada situação, tornando-a eficaz ${ }^{8,9}$.

A pesquisa-ação é composta por quatro etapas principais, são elas: a identificação da situação, em que o pesquisador analisa a situação inicial e percebe a necessidade de mudança, motivando-se a agir; a projetação de soluções, em que se buscam hipóteses de soluções capazes de responder adequadamente às situações identificadas; a implementação de soluções, na qual as opções de soluções são postas em ação, promovendo uma intervenção e a avaliação do procedimento, responsável pela análise dos resultados ${ }^{8}$.

A primeira etapa da pesquisa iniciou-se em 2014, por meio de um estudo realizado com médicos, enfermeiros e cirurgiões-dentistas, que atuavam na Atenção Primária à Saúde (APS) na rede pública do Espírito Santo, para avaliar o perfil de utilização dos serviços ofertados pelo Telessaúde/ES. O Espírito Santo é formado por 78 municípios, sendo que, na época, 67 integravam o Telessaúde ES, totalizando 1.301 profissionais cadastrados nas categorias estudadas, sendo $16 \%$ cirurgiões-dentistas, $33 \%$ enfermeiros e $51 \%$ médicos. Constatou-se a prevalência do sexo feminino entre os profissionais, com tempo de formação acadêmica e tempo de atuação na APS prevalentes no período de 5 a 10 anos. Foi possível identificar uma baixa participação e cadastramento dos cirurgiões-dentistas, em relação ao total dos trabalhadores pesquisados.

Num segundo momento, os integrantes do Telessaúde/ES realizaram outro levantamento, somente com as ESB, para identificar as suas demandas exclusivas e o grau de interesse na criação de um ambiente virtual direcionado. $\mathrm{O}$ interesse na criação do espaço foi unânime entre os trabalhadores participantes do levantamento, sendo que o dia, o horário e os assuntos a serem abordados também foram identificados e definidos.

Utilizou-se, para os dois momentos, um questionário semiestruturado elaborado pela própria equipe da pesquisa, aprovada pelo Comitê de Ética da Universidade Federal do Espírito Santo (CAAE: 30274814.4,0000.5060).

A partir dessa demanda técnica, propôs-se à gestão do Telessaúde/ES o desenvolvimento da Teleodontologia, nomeada posteriormente como Telessaúde Bucal. As atividades iniciaram-se no ano de 2017 dentro dos serviços já ofertados pelo Programa, ou seja, teleconsultoria assíncrona e síncrona, webpalestras, biblioteca virtual, entre outros.

Nesse cenário motivado pelas possíveis integrações sociais e compartilhamento de experiências e conteúdos, pretende-se implantar um ambiente virtual para as ESB, inserido no site do Telessaúde/ES. A estrutura da página do Telessaúde Bucal foi desenvolvida em conjunto com profissionais da área de design e de audiovisual, integrantes da equipe do Telessaúde/ES, por meio de prototipação de telas, não havendo a necessidade de alteração 
imediata do site.

Para o desenvolvimento desse ambiente virtual, priorizou-se o princípio de usabilidade, isto é, a capacidade de um sistema interativo oferecer ao seu usuário a facilidade de aprendizagem e memorização das operações a serem realizadas, promovendo maior rapidez de utilização, menor taxa de erros e maior satisfação do usuário, uma vez que suas metas são alcançadas ${ }^{10}$.

Após a implantação, de fato, do ambiente virtual, será confeccionado um questionário semiestruturado voltado às ESB, para avaliar o grau de satisfação em relação ao Telessaúde Bucal. Além disso, os dados quantitativos referentes à utilização dos serviços de teleconsultoria assíncrona e síncrona, acessos às webpalestras, on-line e off-line, e participação em cursos à distância, serão constantemente monitorados.

\section{RESULTADOS}

Atualmente, o Telessaúde/ES está implantado em 69 municípios do Estado do Espírito Santo e em processo de implantação em nove. Desde o início de suas atividades, em 2012, os profissionais da ESB vêm se cadastrando de maneira progressiva no Programa. Até junho de 2017, havia um total de 551 profissionais cadastrados. No que diz respeito à utilização dos serviços oferecidos de Teleducação, durante o período de 2015 a junho de 2017, houve um crescimento da participação dos profissionais das ESB nas webpalestras on-line (tabela 1).

A partir de 2017, começou-se a realizar, em toda a última sexta-feira de cada mês, webpalestras on-line sobre temas diversos da Odontologia, propostos pelos próprios profissionais. As webpalestras que já foram ministradas são monitoradas e disponibilizadas no site para acesso off-line. "Atendimento odontológico de bebês e crianças na APS" com 324 visualizações, "Principais patologias que acometem a cavidade bucal" com 233 visualizações, "Autoavaliação para melhoria do acesso e da qualidade de atenção básica - PMAQ (saúde bucal)" com 660 visualizações, "Atendimento odontológico ao paciente idoso" com 150 visualizações e "Flúor: dos "8 aos 80?" com 52 visualizações, são as webpalestras de saúde bucal já ministradas em 2017 e disponíveis no canal do YouTube.

Em relação ao serviço de teleconsultoria assíncrona e síncrona, durante o período de 2012 a junho de 2017, 220 teleconsultorias assíncronas foram realizadas por profissionais das ESB, sendo 195 requisições de cirurgiões-dentistas e 25 de Técnicos de Saúde Bucal (tabela 1).

Tabela 1. Frequência numérica de profissionais cadastrados e de utilização dos serviços no Telessaúde/ES

\begin{tabular}{|c|c|c|c|c|c|c|}
\hline \multirow[t]{2}{*}{ Variáveis } & \multicolumn{6}{|c|}{ Frequência (anos) } \\
\hline & 2012 & 2013 & 2014 & 2015 & 2016 & 2017 \\
\hline $\begin{array}{r}\text { Profissionais cadastrados no } \\
\text { Telessaúde/ES }\end{array}$ & 47 & 68 & 174 & 60 & 79 & 123 \\
\hline $\begin{array}{r}\text { Participações on-line nas } \\
\text { webpalestras }\end{array}$ & - & - & - & 19 & 10 & 119 \\
\hline $\begin{array}{r}\text { Número de profissionais que } \\
\text { realizaram Teleconsultorias } \\
\text { assíncronas }\end{array}$ & 5 & 1 & 15 & 12 & 13 & 49 \\
\hline
\end{tabular}


As teleconsultorias síncronas são realizadas juntamente aos especialistas da Universidade Federal do Espírito Santo após solicitação e agendamento prévio. Até junho de 2017, as áreas disponíveis para as teleconsultorias síncronas foram: Saúde Bucal Coletiva, Patologia Bucal e Estomatologia e Odontopediatria. Foram realizadas, neste período, três teleconsultorias.

Atualmente, estão publicadas na Biblioteca Virtual em Atenção Primária à Saúde do Programa Telessaúde Brasil Redes, 14 arquivos de Segunda Opinião Formativa (SOF) de Odontologia, produzidos pelo Telessaúde/ES e disponíveis na página da Tele-educação.

Por fim, a Biblioteca Virtual, localizada na área de Tele-educação, disponibiliza uma pasta com materiais oficiais da área, denominada "Saúde Bucal". São cartilhas, diretrizes, manuais, cadernos, panfletos, protocolos e guias, publicados pela própria equipe de Tele-educação, Ministério da Saúde, Secretarias Estaduais e Municipais de Saúde. A pasta possui 155 visualizações.

\section{Proposta do ambiente virtual do Telessaúde Bucal}

O ambiente virtual do Telessaúde Bucal estará incluso em "Programas Especiais", espaço desenvolvido para a oferta de serviços com enfoque em uma única área de conhecimento. $\mathrm{O}$ acesso a qualquer serviço será realizado com apenas um click, proporcionando maior rapidez de utilização do sistema (figura 1).

A página do Telessaúde Bucal ofertará todo o seu conteúdo em forma de "highlights", que proporciona o destaque automático das informações mais atuais (figura 2).

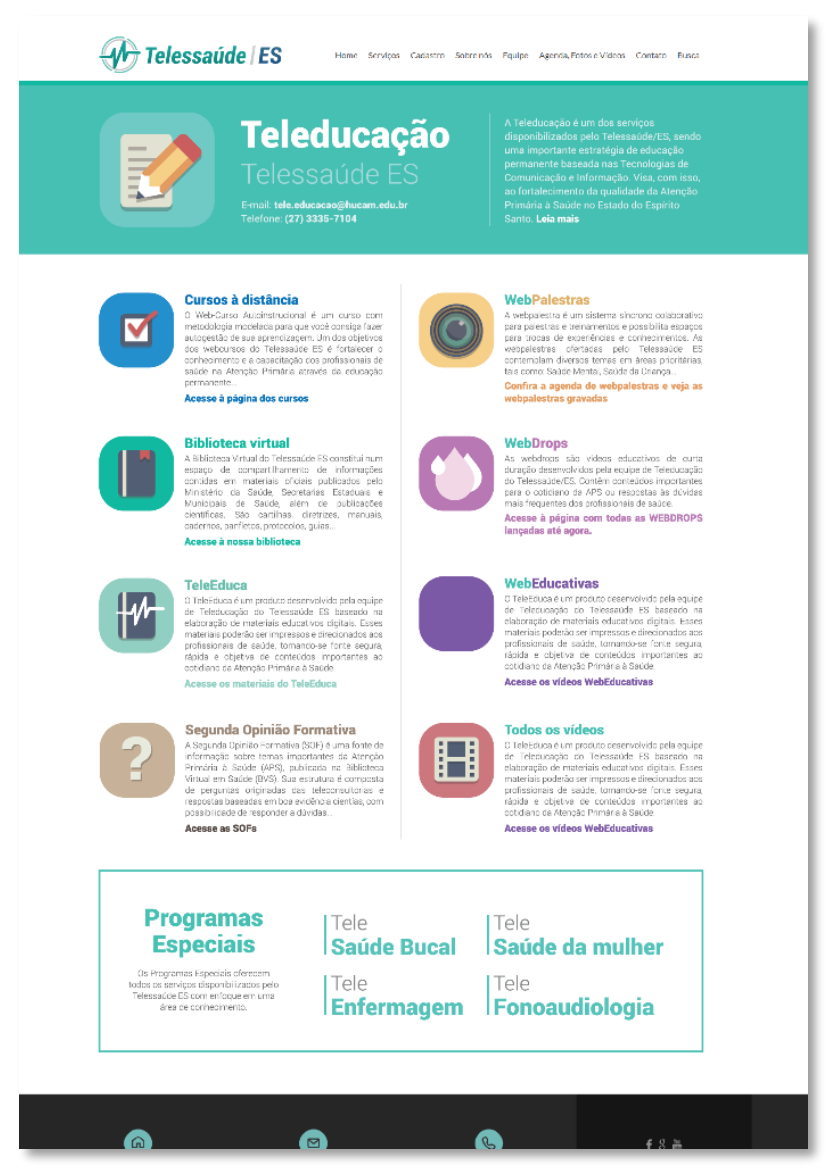

Figura 1. Área de Programas Especiais inserida na página de Teleducação no site do Telessaúde/ES 

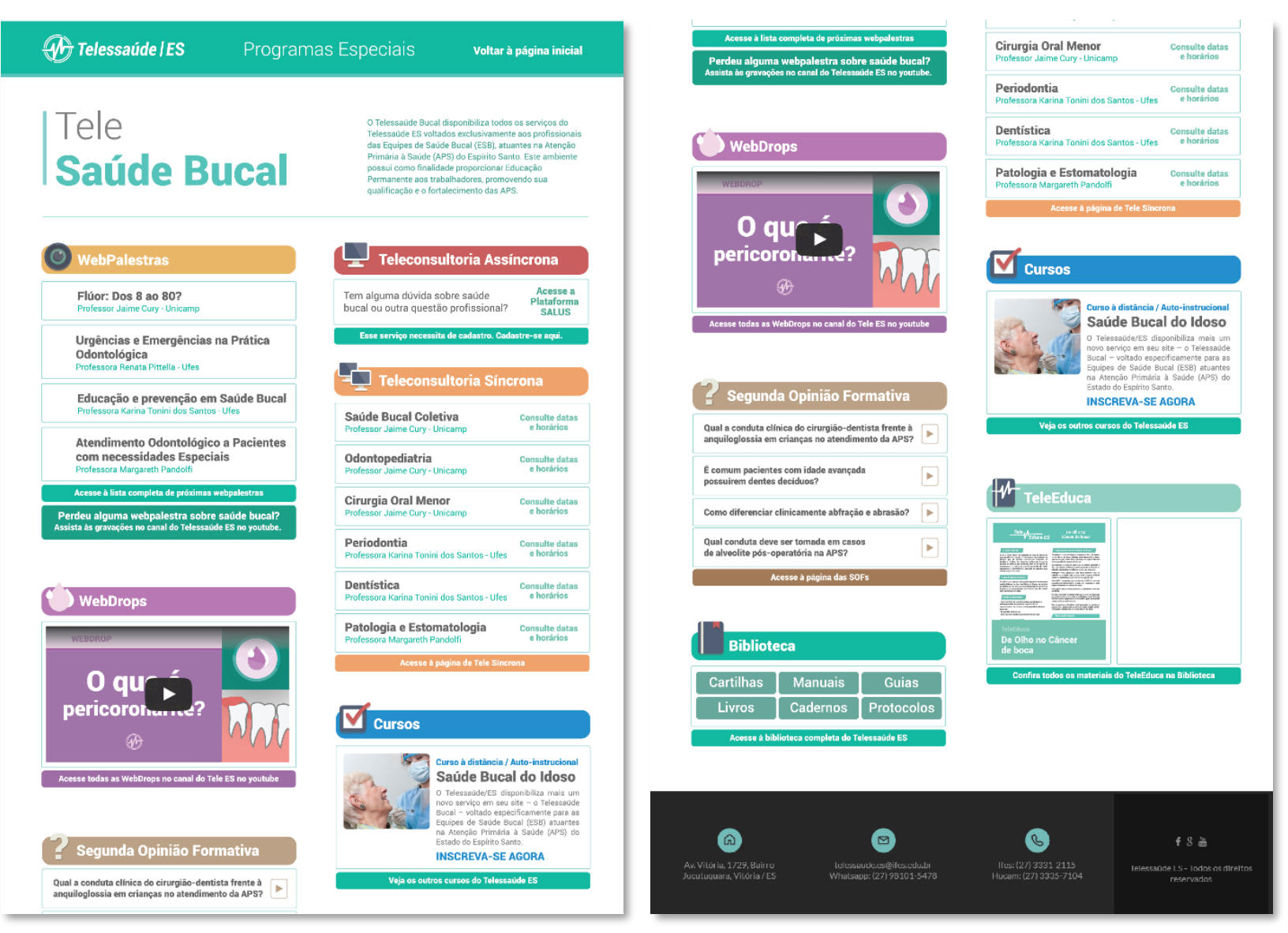

Figura 2. Telas do ambiente virtual Telessaúde Bucal

É importante destacar, que o serviço de webpalestra, diferente da página de Teleducação, se apresentará em um ambiente composto pela agenda de webpalestras, webpalestras gravadas no YouTube e um campo voltado ao público para sugestões de temas para as próximas webpalestras (figura 3).

$\mathrm{O}$ ambiente de cursos à distância apresentará em destaque o curso em andamento e o curso que possuir inscrições abertas. $\mathrm{O}$ profissional poderá, ainda, visualizar os temas de cursos em processo de criação (figura 4).

Também será disponibilizado o serviço TeleEduca, que oferece materiais que abrangem informações de forma rápida, atual e objetiva do cotidiano profissional. Materiais sobre Saúde Bucal da Gestante, Conduta Profissional Frente à Avulsão Dentária, Traumatismo Dentário, Câncer de Boca e Cuidados com as Próteses
Dentárias, estão em processo final de confecção. As Webdrops, vídeos educativos de curta duração, também serão ofertadas, disponibilizando informações importantes aos profissionais.

Finalmente, será proposta a inclusão de novos teleconsultores nas áreas de Cirurgia Oral Menor, Periodontia, Odontogeriatria e Dentística.

\section{DISCUSSÃO}

A cobertura do Telessaúde/ES engloba cerca de $90 \%$ dos municípios do Estado do Espírito Santo, evidenciando que grande parte dos profissionais da ESF do estado é contemplada pelos serviços disponibilizados pelo Programa. As TIC aplicadas à saúde contribuíram para a expansão do Programa Telessaúde no Brasil, que são utilizadas para o aprimoramento da EPS, compartilhamento de 
conhecimentos, pesquisas em saúde e acesso a cuidados em saúde de maneira apropriada, levando

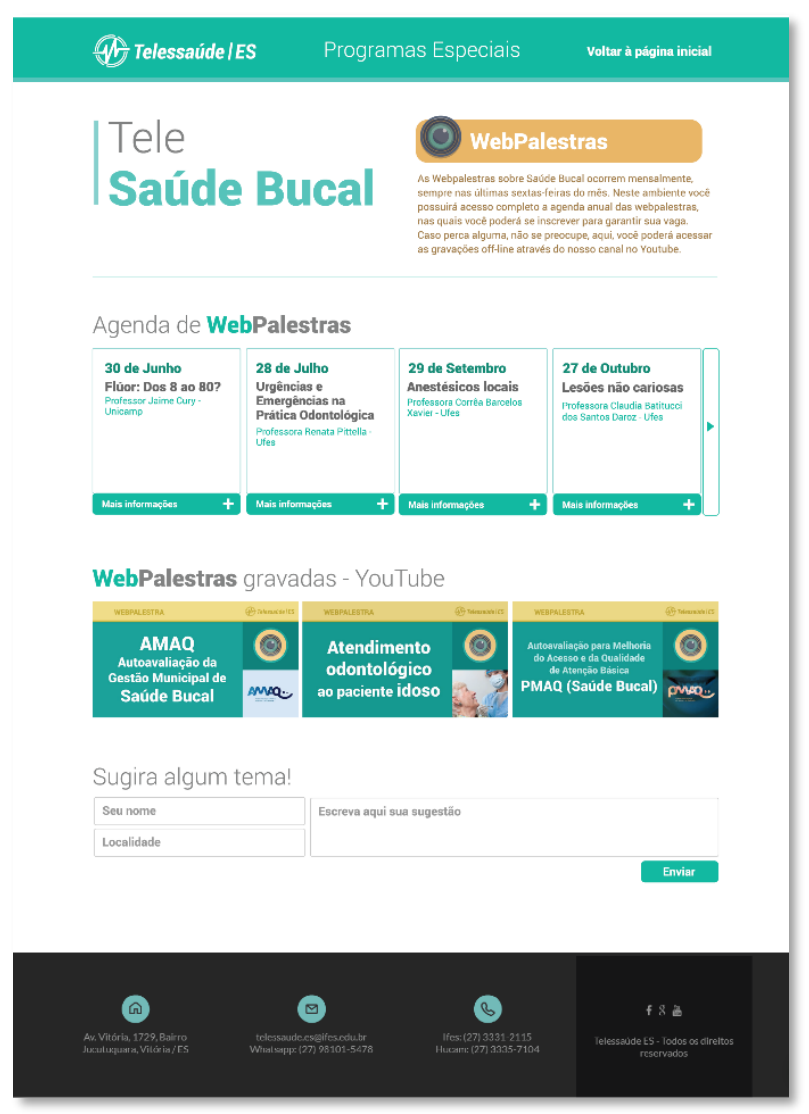

Figura 3. Ambiente virtual do serviço de Webpalestras do Telessaúde Bucal

O Telessaúde é uma ferramenta de ensinoaprendizagem capaz de suprir as necessidades de conhecimento dos profissionais, contribuindo para efetivação do processo de EPS. A utilização de TIC proporciona uma percepção da sociedade atual, mostra a interligação global e promove o acesso contínuo e permanente a toda e qualquer informação ${ }^{12}$.

Neste estudo, verificou-se um aumento progressivo de participação das ESB após o início das atividades do Telessaúde Bucal. Este aumento pode estar relacionado à oferta de atividades específicas de Odontologia no Telessaúde/ES, a partir de 2017, e ao em consideração as necessidades de cada localidade ${ }^{11}$.

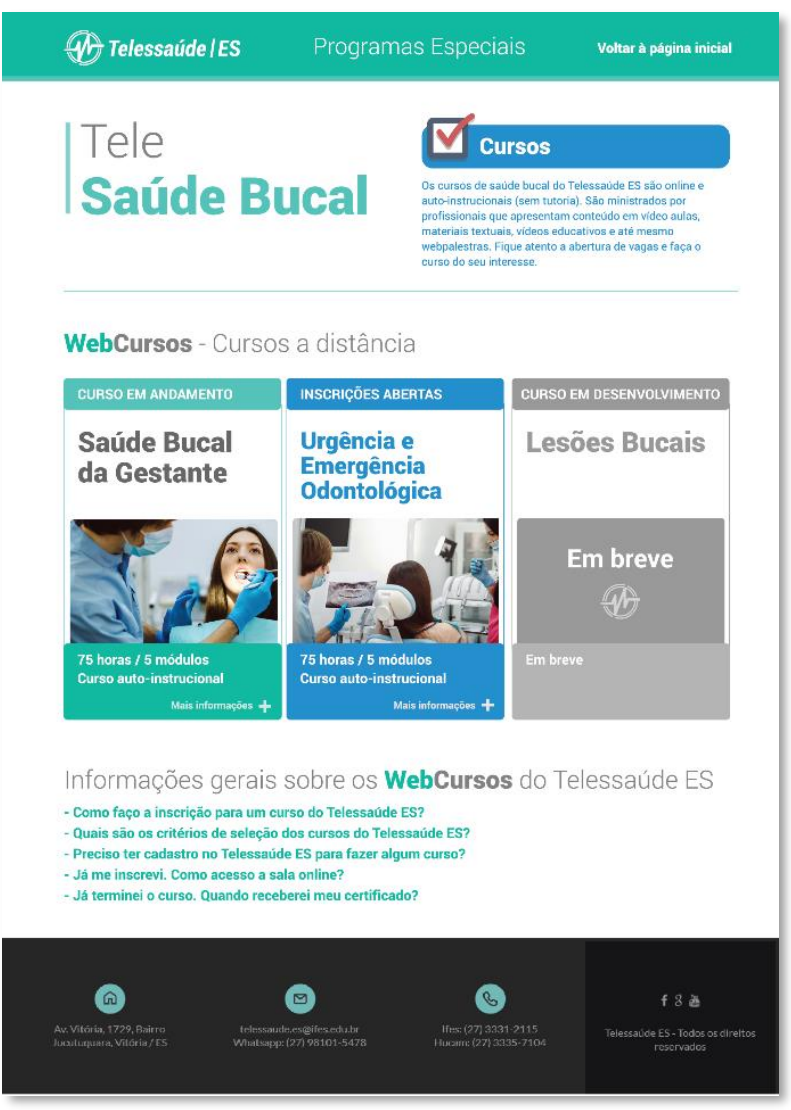

Figura 4. Ambiente virtual do serviço de Cursos do Telessaúde Bucal

planejamento e desenvolvimento dos produtos a partir da necessidade das ESB cadastradas.

Caldarelli e Haddad (2016) ${ }^{13}$ afirmam que a Teleodontologia pode, em consenso com as Diretrizes Curriculares Nacionais (DCN), expandir a inserção das instituições de ensino nas ações de capacitação e EPS dos profissionais atuantes SUS, fortalecendo a integração ensinoserviço. Para esses autores, a difusão da Teleodontologia no território nacional torna-se uma importante estratégia de melhoria da qualidade educacional, já que a Odontologia apresenta papel significativo na saúde da população. 
Entretanto, considerando as 418 ESB implantadas no estado, a participação desses profissionais no Telessaúde/ES ainda é baixa ${ }^{14}$. De acordo com Godoy et al. $(2014)^{15}$, a falta de apoio da gestão, a carência de estratégias para incorporar os serviços nos processos de saúde e a ausência de capacitação dos trabalhadores constituem possíveis causas da baixa participação dos profissionais na utilização dos serviços. Segundo o MS, é competência da gestão promover a integração dos profissionais de saúde com as ações do programa. Outro fator importante é a rotatividade de profissionais nas Unidades Básicas de Saúde (UBS), que também pode possuir impacto na relação do número de profissionais que não fazem uso das ferramentas ${ }^{16}$.

Além disso, alguns profissionais apresentam dificuldade para lidar com as TIC, fazendo-se necessário a superação dessa questão, pois a dificuldade do manejo das ferramentas disponíveis pode limitar o acesso, diminuindo, consequentemente, o impacto do Programa ${ }^{17}$. A ausência de boa infraestrutura tecnológica e conectividade também afetam o seu sucesso ${ }^{15}$.

Com intuito de aumentar a utilização dos serviços oferecidos pelos Programas de Telessaúde, é essencial que as ferramentas disponíveis sejam capazes de promover o interesse do profissional e abordar temas focados na vivência clínica diária da equipe, proporcionando qualidade educacional. Compreende-se que o uso de materiais educativos que priorizem os problemas cotidianos da equipe, e que promovam soluções de problemas reais, são fundamentais para o processo de educação permanente ${ }^{18}$.

Ademais, muitas Equipes de Saúde da Família não possuem uma parte da sua carga horária semanal, conhecida como "horário protegido", destinado ao desenvolvimento de atividades de EPS. A inclusão desse momento, na rotina dos profissionais, poderia garantir a efetiva participação nas atividades do Telessaúde.

É necessário que os trabalhadores e gestores da área de Odontologia compreendam que os produtos do Telessaúde Bucal, ou seja, as teleconsultorias, webconferências, teleEduca, cursos à distância e a própria biblioteca virtual são capazes de proporcionar conhecimento educacional e que essa prática deve ser inserida no seu cotidiano, pois promove benefícios tanto para os próprios profissionais como para a população atendida.

Por esse motivo, mobilizações e ações educativas vêm sendo promovidos pela equipe do Telessaúde/ES com o intuito de diminuir esses impactos e promover a divulgação dos serviços. Em 2014, foram realizados mobilizações e ações educativas in loco, reproduzidos em 2017, resultando em aumentos perceptíveis nos cadastramentos e na utilização dos serviços. Melo et al. (2007), citados por Rendeiro (2012) ${ }^{17}$ afirmam que os envolvidos nas práticas de Telessaúde devem passar por um processo de capacitação sobre o uso das ferramentas e sobre as questões éticas e legais que as envolvem.

Também estão sendo realizadas ações de divulgação do Telessaúde Bucal por meio de reuniões presenciais com os Coordenadores de Saúde Bucal e da Atenção Primária à Saúde do Estado e por meio de reportagens veículadas no jornal do Conselho Regional de Odontologia do Espírito Santo (CRO-ES), no Site do Centro de Ciências da Saúde da UFES, na rede social (Facebook), no aplicativo móvel (WhatsApp) e em e-mails informativos.

Em relato de experiência das ações de Teleodontologia promovidas pelo Programa Telessaúde do Mato Grosso do Sul, Correia et al. $(2014)^{18}$ afirmam que, apesar dos resultados de participação ainda serem baixos, o programa tem se mostrado como importante instrumento de apoio técnico-assistencial, ampliando o acesso 
dos trabalhadores às ações de EPS, inclusive estimulando a interação entre os profissionais do SUS e do Programa.

Informar e conscientizar o profissional que o Telessaúde Bucal é uma ferramenta importante para a promoção de EPS é essencial. Desta forma, torna-se importante a estimulação da aplicabilidade desse serviço desde a formação acadêmica. Haddad et al. (2014) ${ }^{12}$ relatam que, desde 2006, a Faculdade de Odontologia da Universidade de São Paulo (FOUSP) criou o centro de Teleodontologia, que implantou a disciplina de Teleodontologia em cursos de graduação e pós-graduação, com o intuito de estimular os estudantes a utilizarem as TIC, tanto como ambiente educacional como no desenvolvimento de projetos de ação cidadã e desenvolvimento de ações de capacitação e educação permanente para profissionais do SUS.

\section{CONCLUSÃO}

O Telessaúde Bucal é um serviço que poderá promover, por meio de suas ferramentas específicas, informações, conhecimentos, trocas de experiências importantes, EPS para as ESB e melhorar o processo de trabalho.

$\mathrm{O}$ desenvolvimento da área de "Programas Especiais" torna-se fundamental para a divulgação e utilização dos serviços oferecidos especificamente aos trabalhadores das ESB, estimulando, assim, o interesse desses profissionais e proporcionando, consequentemente, o aumento do número de acessos e cadastramentos.

Além disso, esta proposta pode ser aplicada às demais áreas de saúde, contribuindo para o cuidado da população assistida.

\footnotetext{
ABSTRACT

Information and communication technologies for professional training in Dentistry: a Telessaúde/ES proposal

Telehealth/ES, aided by Information and Communication Technologies (ICT), promotes
}

the extension of the Permanent Health Education (EPS) for professionals working at the Family Health Strategy (ESF) of Espírito Santo. The aim of this study was to present a proposal of a virtual environment in Dentistry within Telehealth /ES in order to provide a unique space for Oral Health Teams (ESB) to share information. It is an action-research, which is suitable for proposing solutions for the improvement of access and use of Telehealth/ES tools by ESBs. In 2017, Oral Telehealth initiated its activities in the Telessaúde/ES by providing access to web seminars, delivered every last Friday of the month, asynchronous and synchronous teleconsultation and official materials of the area. The suggested virtual environment of Oral Telehealth will be included in the space of "Special Programs", providing access to any service. It was concluded that Oral Telehealth is a service that can promote knowledge and experience exchange for the implementation of EPS, stimulating the increase in the participation of Dentistry professionals.

Descriptors: Telemedicine. Continued Education. Information Technology. Dentistry.

\section{AGRADECIMENTO}

Ao Programador Visual do Telessaúde/ES Fábio Soares Baptista pela criação dos materiais gráficos.

\section{REFERÊNCIAS}

1. Brasil. Constituição da República Federativa do Brasil. Brasília: Senado Federal, 1988.

2. Brasil. Ministério da Saúde. Portaria $n^{\circ} 1.996$, de 20 de agosto de 2007. Dispõe sobre as diretrizes para a implementação da Política Nacional de Educação Permanente em Saúde.

3. Brasil. Ministério da Saúde. Portaria n ${ }^{\circ} 2.546$, de 27 de outubro de 2011. Diário Oficial da República Federativa do Brasil, Brasília, 27 out. 2011, col 208, p. 50.

4. Sarti TD, Andreão RV, Schimidt MQ, Celestrini J. Teleconsultoria assíncrona como ferramenta de suporte ao trabalho em saúde. Rev Bras Pesq Saúde. 2013 jul-set;15(3):79- 
84.

5. Alkmim MB, Figueira RM, Marcolino MS, Cardoso CS, Pena de Abreu M, Cunha LR et al. Improving patient access to specialized health care: the Telehealth Network of Minas Gerais, Brazil. Bull World Health Organ. 2012 may;90(5):373-8.

6. Haddad AE, Skelton-Macedo MC. Teleodontologia na formação dos profissionais de saúde. In: Mathias I, Monteiro AMV. (Org.). GoldBook: inovação tecnológica em educação e saúde. Rio de Janeiro: EdUERJ; 2012. p. 173-206

7. Caldarelli PG, Haddad AE. Teleodontologia em consonância com as Diretrizes Curriculares Nacionais no desenvolvimento de competências profissionais. Rev ABENO. 2016;16(2):25-32.

8. Dionne H. A pesquisa-ação para o desenvolvimento local. Brasília: Liber Livro Editora; 2007.

9. Gibbs C, Kooyman B, Marks K, Burns J. Mapping the roadmap: using action research to develop an online referencing tool. Acad Librarian. 2015;41:422-8.

10. Macedo MKB. recomendações de acessibilidade e usabilidade para ambientes virtuais de aprendizagem voltados para o usuário idoso [dissertação]. Florianópolis: Universidade Federal de Santa Catarina; 2007.

11. Damasceno FR, Reategui EB, Harzheim E, Schmitz CAA, Epstein D. Um estudo sobre o emprego da mineração textual para classificação de teleconsultorias no contexto do Projeto Telessaúde-RS. Rev Eletron Comum Inf Inov Saúde. 2016 abr-jun;10(2):16.

12. Haddad AE, Bönecker M, Skelton-Macedo MC. Research in the Field of Health, Dentistry, Telehealth and Teledentistry. Braz Oral Res. 2014;28(1):1-2.
13. Caldarelli PG, Haddad AE. Teleodontologia em consonância com as Diretrizes Curriculares Nacionais no desenvolvimento de competências profissionais. Rev ABENO. 2016;16(2):25-32.

14. Nota Técnica do DAB [Internet]. Nota Técnica. [Acesso em 18/06/2017]. Disponível em: http://dab2.saude.gov.br/sistemas/nota tecnica/frmListaMunic.php.

15. Godoy SCB, Guimarães EMP, Assis DSS. Avaliação da capacitação dos enfermeiros em unidades básicas de saúde por meio da teleenfermagem. Esc Anna Nery. 2014 jan.mar.;18(1):148-155.

16. Marcolino MS, Alkmim MB, Assis TGP, Sousa LAP, Ribeiro ALP. Teleconsultorias no apoio à atenção primária à saúde em municípios remotos no estado de Minas Gerais, Brasil. Rev Panam Salud Publica. 2014;35(5/6):345-52.

17. Rendeiro MMP, Vieira C, Figueiredo DLTM. Infocultura: uma barreira de acesso à educação permanente? In: Mathias I, Monteiro AMV. (Org.). GoldBook: inovação tecnológica em educação e saúde. Rio de Janeiro: EdUERJ; 2012. p. 832-845. [Acesso em 18/06/2017]. Disponível em: http://www.telessaude.uerj.br/resource/goldb ook/pdf/48.pdf.

18. Correia ADMS, Dobashi BF, Gonçalves CCM, Monreal VRFD, Nunes EA, Haddad $\mathrm{PA}$, et al. Teleodontologia no programa nacional telessaúde Brasil redes: relato da experiência em Mato Grosso do Sul. Rev ABENO. 2014;14(1):17-29.

\section{Correspondência para:}

Karina Tonini dos Santos Pacheco

e-mail: kktonini@yahoo.com.br

Departamento de Medicina Social UFES

Av. Marechal Campos, 1468 Maruípe

29090-041 Vitoria/ES 